\title{
Resultados de la osteotomía y la osteosíntesis rígida en la consolidación viciosa de falange
}

\author{
Ignacio Rellán, Luis Ferreira, Pablo De Carli, Gerardo L. Gallucci, Agustín Donndorff, Ezequiel E. Zaidenberg, \\ Jorge G. Boretto \\ Sector de Cirugía de Mano y Miembro Superior, Instituto de Ortopedia y Traumatología "Prof. Dr. Carlos E. Ottolenghi", \\ Hospital Italiano de Buenos Aires, Ciudad Autónoma de Buenos Aires, Argentina
}

\begin{abstract}
RESUMEN
Objetivo: Evaluar los resultados y las complicaciones de una serie de pacientes con consolidación viciosa de falange. Materiales y Métodos: Se realizó un estudio retrospectivo que incluyó a pacientes con consolidación viciosa de falange tratados mediante osteotomía en el sitio de la deformidad, estabilizados con osteosíntesis rígida. Se registró la movilidad de los dedos. La valoración subjetiva consistió en el puntaje DASH y una escala analógica visual para el dolor en reposo, en actividad y la función. Resultados: Doce pacientes (13 falanges) cumplieron los criterios de inclusión. El tiempo promedio entre la lesión inicial y la osteotomía fue 14 meses y el seguimiento promedio, 34 meses. La movilidad final promedio en flexo-extensión fue $89^{\circ}$ metacarpofalángica, $74^{\circ}$ interfalángica proximal, $54^{\circ}$ interfalángica distal y la distancia pulpejo-palma, $3 \mathrm{~mm}$. El puntaje promedio de la escala analógica visual en reposo fue 0; en actividad, 1 y la función promedio fue de 7 puntos; el puntaje DASH promedio fue 6 . No hubo casos de seudoartrosis. Cinco pacientes requirieron el retiro de la placa. Los pacientes con osteotomía articular no presentaron signos de artrosis. Conclusiones: La osteotomía de falange en el sitio de la deformidad es un procedimiento eficaz con un buen resultado objetivo y subjetivo a corto plazo. La utilización de placas y tornillos conlleva una tasa más alta de complicaciones; por lo tanto, los pacientes deben ser advertidos sobre la posibilidad de una segunda intervención quirúrgica.
\end{abstract}

Palabras clave: Osteotomía; falange; consolidación viciosa.

Nivel de Evidencia: IV

\section{Outcomes of osteotomy and rigid internal fixation treatment for the correction of phalangeal malunions}

\begin{abstract}
Objective: To present the results and complications of a series of phalangeal malunion patients. Materials and Methods: $\mathrm{A}$ retrospective study was conducted on phalangeal malunion patients treated with an osteotomy at the deformity site and stabilized with rigid internal fixation. Postoperative mobility of the digit was recorded. The subjective assessment used the Disabilities of the Arm, Shoulder and Hand (DASH) score and a Visual Analogue Scale (VAS) to rate their function and pain at rest and during activity. Results: Twelve patients (13 phalanges) met the inclusion criteria. The average time between fracture and osteotomy was 14 months and the mean follow-up was 34 months. Final mobility in flexo-extension was: 89 degrees for metacarpophalangeal joint, 74 degrees proximal interphalangeal joint, 54 degrees distal interphalangeal joint. The average VAS pain score was 0 at rest and 1 during activity, and the average function was 7 points. The average DASH score was 6 . There were no cases of nonunion. Five patients required plate removal. No patients with articular osteotomy presented signs of Osteoarthritis. Conclusions: Phalangeal osteotomy at the deformity site is an effective procedure with good objective and subjective short-term outcomes. Patients should be warned about the possibility of a second procedure in cases of plate fixation due to a higher complication rate associated with plate and screw fixations.
\end{abstract}

Key words: Osteotomy; phalanx; malunion.

Level of Evidence: IV

Recibido el 18-12-2018. Aceptado luego de la evaluación el 3-11-2019 • Dr. IGNACIO RELLÁN • ignacio.rellan@ hospitalitaliano.org.ar (ID

Cómo citar este artículo: Rellán I, Ferreira L, De Carli P, Gallucci GL, Donndorff A, Zaidenberg EE, Boretto JG. Resultados de la osteotomía y la osteosíntesis rígida en la consolidación viciosa de falange. Rev Asoc Argent Ortop Traumatol 2020;85(2):125-132. https://doi.org/10.15417/issn.1852-7434.2020.85.2.932 


\section{INTRODUCCIÓN}

La consolidación viciosa en las falanges puede tener diferentes componentes, como rotación, angulación, acortamiento, escalón articular o una combinación de ellos. Esto puede producir una limitación en el cierre del puño debido al entrecruzamiento de los dedos, el desequilibrio en el aparato extensor, la pérdida de fuerza de puño y el dolor articular.

Se ha debatido sobre las ventajas y desventajas de tratar los defectos rotacionales en el sitio de la deformidad o a nivel metacarpiano. Corregir la consolidación viciosa de falange en el sitio de la fractura inicial permite realizar procedimientos asociados, como tenólisis o capsulotomías y, a la vez, tratar, en forma más eficiente, las deformidades rotacionales, angulares, combinadas, y constituye la única manera de tratar las deformidades articulares. ${ }^{2}$ Por el contrario, la corrección a nivel metacarpiano es técnicamente más sencilla y evitaría adherencias tendinosas. ${ }^{3}$ Sin embargo, este tipo de corrección se puede aplicar solo a deformidades rotacionales y estudios cadavéricos han demostrado que esta última se limita a deformidades rotacionales $<15^{\circ} .{ }^{4}$

Con excepciones, ${ }^{2,5,6}$ la bibliografía disponible hace referencia a series pequeñas, tratadas con osteotomías correctivas a nivel metacarpiano $\mathrm{o}^{7-9} \mathrm{o}$ con osteosíntesis no rígida y un seguimiento acotado. ${ }^{10,11}$

El objetivo de este estudio fue evaluar los resultados y las complicaciones de una serie de pacientes con consolidación viciosa de falange tratados mediante osteotomía en el sitio de la deformidad y fijación interna rígida.

\section{MATERIALES Y MÉTODOS}

Para el propósito de este estudio retrospectivo, se revisaron los registros médicos y se invitó a un examen de seguimiento a pacientes con consolidación viciosa de falange tratados en nuestra institución.

Los criterios de inclusión fueron: 1) edad >18 años, 2) consolidación viciosa de falange, 3) tratamiento con osteotomía en el sitio de la deformidad y 4) estabilización mediante placas o tornillos. Se excluyó a los pacientes $<18$ años y a los tratados sin fijación interna rígida.

Dentro de las variables por estudiar, se registraron el sexo y la presencia de artrosis (en aquellos pacientes con deformidades articulares) como variables cualitativas dicotómicas. El hueso y el dedo involucrados, el tipo de consolidación viciosa, los antecedentes y el tipo de cirugía previa, el abordaje empleado, la osteosíntesis utilizada, los gestos quirúrgicos asociados, las intervenciones quirúrgicas posteriores y las complicaciones posoperatorias se registraron como variables cualitativas nominales. También se consideraron como variables cuantitativas continuas la edad, el tiempo transcurrido entre la fractura y la osteotomía, y el tiempo de seguimiento hasta la fecha de comenzar la revisión. El tipo de deformidad fue clasificado como angular, rotacional, articular o una combinación de ellos. Se definió complicación a cualquier desviación de la evolución posoperatoria esperable que provocase dolor, limitación funcional o requiriese tratamiento adicional (quirúrgico o no quirúrgico). Las complicaciones fueron divididas utilizando la clasificación de complicaciones quirúrgicas propuesta por Dindo y cols. ${ }^{12}$ La experiencia de los cirujanos tratantes fue clasificada según la clasificación propuesta por Tang y cols.. ${ }^{13}$ Nivel I: cirujano no especialista, Nivel II: especialista con menos de 5 años de experiencia, Nivel III: especialista con más de 5 años de experiencia, Nivel IV: especialista altamente experimentado, Nivel V: experto.

La corrección de los defectos angulares laterales se planificó midiendo el ángulo hacia radial o cubital para llevar a un eje neutro $\left(0^{\circ}\right)$. La corrección de las angulaciones anteroposteriores se planificó con una radiografía de perfil midiendo el ángulo sea volar o dorsal para llevar a una corrección del eje a $0^{\circ}$.

Las deformidades rotacionales fueron evaluadas clínicamente mediante el entrecruzamiento de los dedos y en aquellos pacientes que no conseguían cerrar el puño, considerando el paralelismo de las placas ungueales. En las deformidades articulares, el objetivo fue lograr una reducción anatómica.

\section{Evaluación posoperatoria}

Los pacientes fueron contactados y citados para un nuevo control, en el que se los evaluó objetivamente registrando la movilidad del dedo afectado en flexo-extensión a nivel metacarpofalángico, interfalángico proximal e interfalángico distal, utilizando un goniómetro manual apoyado en el dorso del dedo y solicitándole al paciente que realice una flexión máxima y una extensión máxima. Se registró, además, la distancia pulpejo-palma pidiéndole al paciente que cierre el puño acercando los dedos a la palma tanto como le sea posible y midiendo con una regla la distancia remanente entre el pulpejo del dedo afectado y el pliegue palmar distal. Para la evaluación subjetiva se utilizó el puntaje DASH (Disabilities of the Arm, Shoulder and Hand) y la escala analógica visual (EAV) de 0 a 10 
para dolor en reposo (donde 0 indica ausencia de dolor y 10, el máximo dolor experimentado), en actividad (donde 0 indica ausencia de dolor y 10, el máximo dolor experimentado) y para la función (donde 0 indica discapacidad completa y 10, una función completa). Aquellos pacientes que no pudieron o se negaron a concurrir para un nuevo examen presencial fueron evaluados solo en forma subjetiva, por correo electrónico o por teléfono (DASH y EAV) y se registraron como seguimiento subjetivo exclusivamente.

\section{Análisis estadístico}

Las variables continuas se presentan como promedio y desviación estándar, y las variables nominales y ordinales, como porcentajes.

\section{RESULTADOS}

En un período de ocho años (2008-2016), 20 falanges (19 pacientes) fueron operadas por una consolidación viciosa, en nuestra institución. Un paciente fue excluido, porque se perdió en el seguimiento; dos fueron excluidos, porque no se les había realizado un método de fijación rígida y tres, porque el seguimiento era $<6$ meses y rechazaron concurrir a un nuevo control presencial. Doce pacientes (13 falanges) cumplieron los criterios de inclusión (Tabla 1). Se incluyó a siete hombres y cinco mujeres, con una edad promedio de 34 años (rango 19-60, desviación estándar 13.3). El tiempo promedio entre la lesión inicial y la osteotomía fue de 14 meses (rango 1-96, desviación estándar 26.2). Cuatro pacientes (uno con fracturas en dos falanges) habían sido operados en otra institución, donde se les colocaron agujas de Kirschner percutáneas para estabilizar la fractura y la reducción fue insuficiente. Los ocho pacientes restantes fueron tratados inicialmente mediante inmovilización con yeso.

Tabla 1. Datos demográficos

\begin{tabular}{|c|c|c|c|c|c|c|c|}
\hline Caso & Edad & $\begin{array}{c}\text { Tiempo entre la fractura } \\
\text { y la osteotomía } \\
\text { (meses) }\end{array}$ & Hueso involucrado & Deformidad & $\begin{array}{c}\text { Artrosis } \\
\text { final }\end{array}$ & $\begin{array}{l}\text { Cirugía } \\
\text { previa }\end{array}$ & Fijación \\
\hline 1 & 34 & 36 & Primera falange, $4^{\circ}$. dedo & Rotacional & N/A & Sí & Placa \\
\hline 2 & 28 & 96 & Primera falange, $4^{\circ}$. dedo & $15^{\circ}$ cubital & N/A & No & Placa \\
\hline 3 & 60 & 3 & Primera falange, $3^{\text {er }}$. dedo & Articular & Sí & No & Tornillos \\
\hline 4 & 37 & 1 & Primera falange, $3^{\text {er }}$. dedo & Articular & Sí & No & Tornillos \\
\hline 5 & 22 & 9 & Primera falange, $3^{\text {er }}$. dedo & Articular & Sí & No & Placa \\
\hline 6 & 56 & 2 & Primera falange, $3^{\text {er }}$. dedo & Rotacional & N/A & No & Placa \\
\hline 7 & 23 & 2 & Segunda falange, $4^{\circ}$. dedo & $10^{\circ}$ cubital & N/A & No & Placa \\
\hline \multirow[t]{2}{*}{8} & \multirow[t]{2}{*}{28} & 9 & Primera falange, $3^{\text {er }}$. dedo & $10^{\circ}$ radial & N/A & Sí & Placa \\
\hline & & 9 & Primera falange, $4^{\circ}$. dedo & $13^{\circ}$ cubital & N/A & Sí & Placa \\
\hline 9 & 40 & 1,5 & Primera falange, $3^{\text {er }}$. dedo & $20^{\circ}$ cubital & N/A & No & Placa \\
\hline 10 & 19 & 10 & Primera falange, $4^{\circ}$. dedo & $10^{\circ}$ cubital & N/A & Sí & Placa \\
\hline 11 & 23 & 7 & Primera falange, $2^{\circ}$. dedo & Rotacional & N/A & No & Tornillos \\
\hline 12 & 41 & 1.5 & Primera falange, $2^{\circ}$. dedo & Articular & Sí & No & Placa \\
\hline Promedio & 34 & 12 & & & & & \\
\hline
\end{tabular}


El seguimiento objetivo (movilidad, EAV y DASH) promedio desde la osteotomía fue de 34 meses (rango 6-62) (Figura). Tres pacientes se negaron o no pudieron concurrir a un nuevo control presencial, por lo que solo se pudo actualizar su evaluación subjetiva (DASH y EAV) por correo electrónico y se tuvieron en cuenta los últimos registros de movilidad que figuraban en la historia clínica. En 12 casos, la falange involucrada fue la primera y, en un caso, la segunda. La indicación de osteotomía fue por deformidad en varo-valgo (6 falanges), por deformidad articular (4 falanges) y por defectos rotacionales (3 falanges).

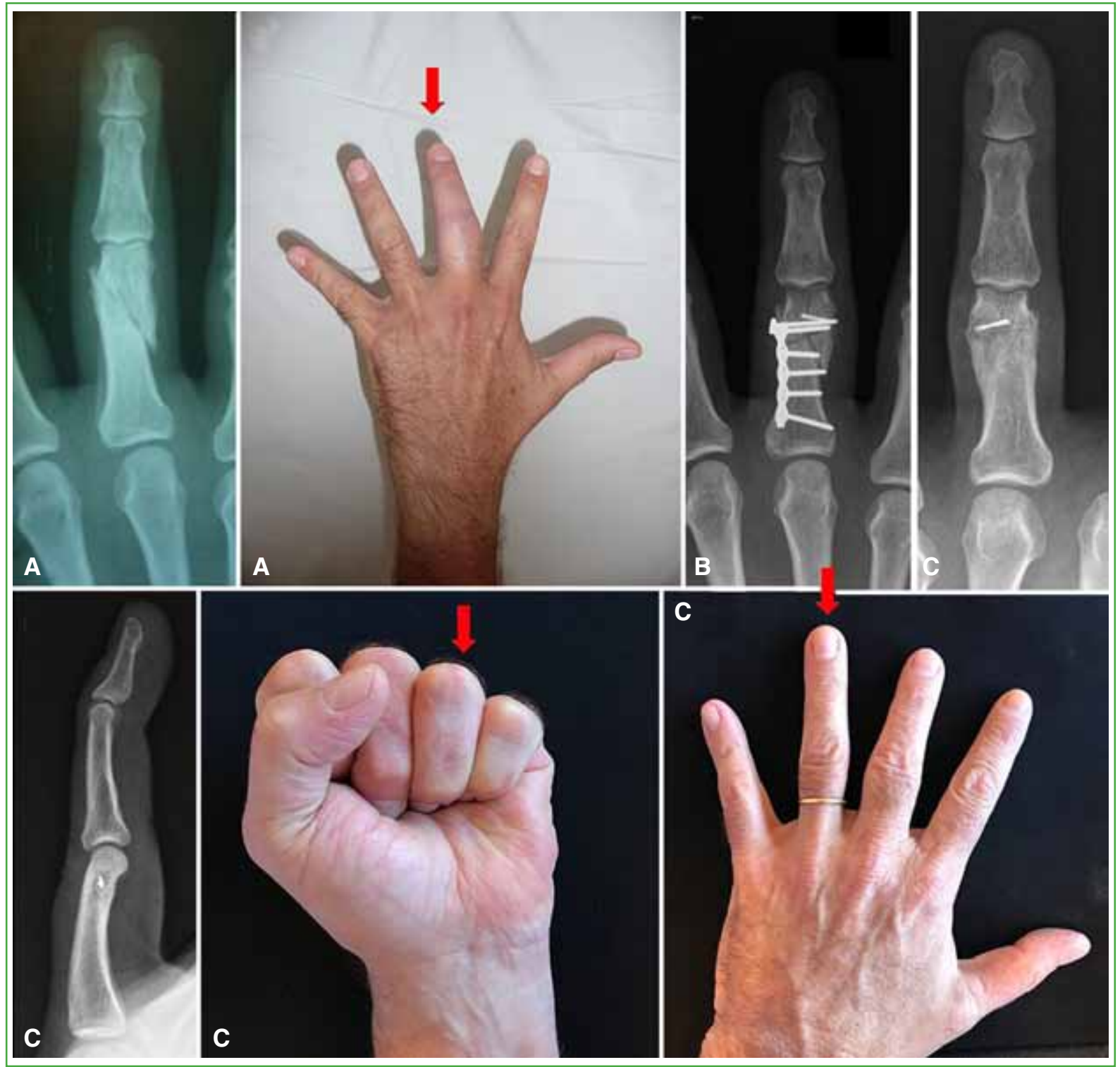

Figura. A. Radiografía e imagen clínica de una consolidación viciosa combinada de dos meses de evolución. B. Radiografía posoperatoria. C. Resultado a los 62 meses del retiro de la osteosíntesis y una tenólisis asociada por adherencias. Se puede observar el remanente de una mecha en la falange, que no ocasionó molestias. 


\section{Técnica quirúrgica}

En 12 casos, la vía de abordaje seleccionada fue dorsal y, en el caso restante, lateral; todos los procedimientos fueron realizados por tres cirujanos diferentes con un nivel de experiencia IV. En cuatro falanges, la osteotomía fue intrarticular por compromiso de esta y extrarticular en las nueve falanges restantes. Se utilizaron como métodos de fijación placas bloqueadas ( 8 falanges), placas no bloqueadas ( 2 falanges) y tornillos interfragmentarios ( 3 falanges). En tres pacientes, fue necesario agregar injerto óseo autólogo; en dos pacientes, se realizó una artrólisis y, en uno, una tenólisis de tendón extensor como gesto quirúrgico asociado (Tabla 2).

Tabla 2. Resultados funcionales

\begin{tabular}{|c|c|c|c|c|c|c|c|c|c|c|c|c|}
\hline Caso & $\begin{array}{l}\text { Seguimiento } \\
\text { subjetivo }\end{array}$ & $\begin{array}{l}\text { Seguimiento } \\
\text { objetivo }\end{array}$ & $\begin{array}{l}\text { Tenólisis/ } \\
\text { artrólisis }\end{array}$ & $\begin{array}{c}\text { EAV } \\
\text { en } \\
\text { reposo }\end{array}$ & $\begin{array}{c}\text { EAV } \\
\text { en } \\
\text { actividad }\end{array}$ & $\begin{array}{l}\text { EAV } \\
\text { función }\end{array}$ & $\begin{array}{l}\text { DASH } \\
\text { posope- } \\
\text { ratorio }\end{array}$ & $\begin{array}{c}\text { Movilidad } \\
\text { MTCF }\end{array}$ & $\begin{array}{l}\text { Movilidad } \\
\text { IFP }\end{array}$ & $\begin{array}{c}\text { Movilidad } \\
\text { IFD }\end{array}$ & $\begin{array}{c}\text { DPP } \\
(\mathrm{mm})\end{array}$ & $\begin{array}{l}\text { Complica- } \\
\text { ciones }\end{array}$ \\
\hline 1 & 54 & 54 & Sí & 0 & 0 & 8 & 0 & 90 & 80 & 55 & 0 & $\begin{array}{c}\text { Retiro del } \\
\text { material de } \\
\text { osteosíntesis }\end{array}$ \\
\hline 2 & 66 & 66 & No & 0 & 2 & 8 & 2 & 120 & 124 & 55 & 0 & No \\
\hline 3 & 51 & 31 & Sí & 0 & 0 & 8 & 0 & 116 & 80 & 86 & 0 & No \\
\hline 4 & 100 & 15 & No & 0 & 3 & 6 & 20 & 95 & 105 & 70 & 0 & No \\
\hline 5 & 48 & 6 & No & 0 & 0 & 6 & 5 & 120 & 80 & 61 & 0 & $\begin{array}{c}\text { Retiro del } \\
\text { material de } \\
\text { osteosíntesis }\end{array}$ \\
\hline 6 & 62 & 62 & No & 0 & 0 & 9 & 0 & 100 & 85 & 40 & 0 & $\begin{array}{c}\text { Retiro del } \\
\text { material de } \\
\text { osteosíntesis }\end{array}$ \\
\hline 7 & 49 & 49 & No & 0 & 2 & 8 & 10 & 90 & 85 & 65 & 0 & No \\
\hline \multirow[t]{2}{*}{8} & \multirow[t]{2}{*}{26} & \multirow[t]{2}{*}{26} & No & 0 & 1 & 7 & 0 & 60 & 78 & 45 & 10 & $\begin{array}{c}\text { Retiro del } \\
\text { material de } \\
\text { osteosíntesis }\end{array}$ \\
\hline & & & No & 0 & 1 & 7 & 0 & 32 & 80 & 50 & 10 & $\begin{array}{c}\text { Retiro del } \\
\text { material de } \\
\text { osteosíntesis }\end{array}$ \\
\hline 9 & 62 & 62 & No & 2 & 4 & 6 & 18 & 88 & 10 & 10 & 15 & $\begin{array}{c}\text { Pérdida } \\
\text { de reducción }\end{array}$ \\
\hline 10 & 8 & 8 & No & 0 & 1 & 7 & 6 & 90 & 24 & 42 & 0 & Rigidez \\
\hline 11 & 36 & 16 & Sí & 0 & 0 & 7 & 2 & 80 & 85 & 60 & 0 & No \\
\hline 12 & 16 & 16 & No & 0 & 0 & 8 & 16 & 70 & 50 & 60 & 25 & $\begin{array}{c}\text { Falla de la } \\
\text { osteosíntesis }\end{array}$ \\
\hline Pro- & 48 & 34 & & 0 & 1 & 7 & 6 & 89 & 74 & 54 & 3 & \\
\hline
\end{tabular}

EVA = escala analógica visual, DASH (Disabilities of the Arm, Shoulder and Hand), MTCF = metacarpofalángica, IFP = interfalángica proximal,

IFD = interfalángica distal, DPP = distancia pulpejo-palma. La movilidad MTCF, IFP e IFD se expresa como movimiento activo total (flexión-extensión) en grados.

\section{Período posoperatorio}

Los pacientes fueron inmovilizados por 7-14 días para luego comenzar con rehabilitación supervisados por una terapista ocupacional, excepto aquellos que fueron sometidos a tenólisis/artrólisis quienes comenzaron dentro de la primera semana. 
La movilidad final promedio en flexo-extensión fue de $89^{\circ}$ metacarpofalángica (rango 32-120), $74^{\circ}$ interfalángica proximal (rango 10-124), 54 interfalángica distal (rango 10-86) y la distancia pulpejo-palma, $4 \mathrm{~mm}$ (rango $0-25$ ). El puntaje promedio de la EAV fue de 0 (rango 0-2) en reposo, 1 (rango 0-4) en actividad y la función promedio fue de 7 (rango 6-9); el puntaje DASH promedio fue de 6 (rango 0-20).

En todos los pacientes, se corrigió la deformidad y se logró la consolidación entre las 7 y 8 semanas después de la cirugía.

\section{Complicaciones}

En cuatro pacientes (5 falanges), fue necesario extraer la placa. En todos los casos, el implante provocaba dolor. En tres pacientes, se asoció una tenólisis del aparato extensor en zona 4, en el momento de la extracción, para aumentar el rango de movilidad y, en un caso, fue necesario efectuar una tenosinovectomía y una tenólisis de los tendones flexores por adherencia de la fractura inicial. En promedio la extracción se realizó a los cuatro meses de la cirugía (rango 3-7).

Dos pacientes sufrieron pérdida de reducción posoperatoria temprana. En un caso, se procedió a una nueva reducción y se aumentó la fijación con clavijas y, en el otro, la pérdida de reducción se debió a falla del implante y se revisó la osteosíntesis colocando una nueva placa. Un paciente presentó rigidez interfalángica proximal (rango en flexo-extensión $24^{\circ}$ ), pero rechazó someterse a un nuevo procedimiento quirúrgico. Todas estas complicaciones fueron clasificadas como IIIa (complicaciones que requieren resolución quirúrgica). Los pacientes con osteotomía articular no presentaron signos de artrosis en las radiografías solicitadas al final de su seguimiento. Ninguno de los tres pacientes de esta serie tratados exclusivamente con tornillos interfragmentarios refirieron molestias asociadas a la osteosíntesis ni complicaciones.

\section{DISCUSIÓN}

Las consolidaciones viciosas de falange fueron tratadas inicialmente mediante una osteotomía a nivel metacarpiano fundamentado en el principio de evitar el sitio de la deformidad disminuyendo así la incidencia de adherencias. ${ }^{7-9}$ Sobre la base de los buenos resultados y la baja incidencia de rigidez, Buchler y cols. ${ }^{2}$ recomendaron el tratamiento de dichas deformidades mediante osteotomía en el sitio de la deformidad. Publicaron una serie 59 osteotomías con diferentes medios de estabilización que incluyeron cerclajes de alambre, clavijas de Kirschner, placas, tornillos o combinaciones de ellas. Reportan que el rango de movilidad mejoró en todos los casos, salvo en tres. Sin embargo, no hay datos preoperatorios ni posoperatorios del rango de movilidad. El seguimiento promedio comunicado es de 11 meses, razón por la cual desconocemos las posibles complicaciones a largo plazo de esta serie.

van der Lei y cols. ${ }^{14}$ reportaron una serie de nueve osteotomías en el sitio de la deformidad estabilizadas ( 3 con cerclaje de alambre, 4 con clavijas y 2 con placas y tornillos). En siete casos, se logró una corrección satisfactoria, pero dos pacientes debieron ser operados nuevamente por falta de corrección de la deformidad inicial, y se logró la corrección en solo uno de ellos. Los autores reportan que no hubo pérdida de la movilidad preoperatoria; sin embargo, no informan qué rango de movilidad tenían los pacientes. Podemos inferir, de acuerdo con la evaluación de nuestra serie, que los pacientes no tenían una movilidad preoperatoria completa. Trumble y cols. ${ }^{5}$ publicaron una serie de 11 osteotomías extrarticulares estabilizadas con placas dorsales y un seguimiento de 35 meses. En promedio mejoró el rango de movilidad interfalángica proximal e interfalángica distal. Sin embargo, debieron retirar el 100\% de las placas por molestias. Lamentablemente no informan el tiempo transcurrido entre la osteotomía y el retiro de la osteosíntesis, dato que hubiese sido interesante conocer para comparar con nuestra casuística.

Potenza y cols. ${ }^{6}$ reportaron una serie retrospectiva de 24 osteotomías con osteosíntesis rígida (6 con tornillos y 18 con placas y tornillos) y un seguimiento promedio de 24 meses. Dos pacientes debieron ser sometidos a una segunda intervención quirúrgica para realizar una tenólisis/artrólisis más el retiro del implante. Dos de las cuatro osteotomías intrarticulares presentaron signos leves de artrosis al final del seguimiento. Todos los pacientes lograron un 30\% de mejoría de la movilidad previa sin ningún caso de rigidez o molestias de la osteosíntesis.

Aquí se presenta una serie de 13 osteotomías (12 pacientes) de falange realizadas en el sitio de la deformidad, estabilizadas con osteosíntesis rígida, y un seguimiento objetivo promedio de 34 meses y un seguimiento subjetivo promedio de 48 meses. A pesar de que la tasa de retiro de placas en nuestra serie (50\%; 5 de 10 placas colocadas) es inferior a la comunicada por Trumble 5 (100\%), continúa siendo elevada. Sin embargo, el dolor asociado al implante en las falanges no es un cuadro que debe considerarse como una situación infrecuente, por lo que se debe conversar con el paciente sobre la alta probabilidad de realizar una extracción durante el seguimiento posoperatorio. 
La pérdida de movilidad y la seudoartrosis definitivamente no son una complicación frecuente de este procedimiento, tal como lo demuestran nuestros resultados y los de otros autores antes citados. ${ }^{2,5,6,14}$ No obstante, para asegurarse de mantener o mejorar la movilidad preoperatoria, es esencial utilizar una osteosíntesis rígida que permita una rehabilitación activa temprana. . $^{15-17}$

Entre las fortalezas de nuestro estudio, podemos enumerar el considerable tamaño de la serie teniendo en cuenta la esporádica frecuencia con que se realiza este procedimiento.

La inclusión de osteotomías intrarticulares y extrarticulares, y el carácter retrospectivo de esta muestra constituyen su principal debilidad.

Concluimos, por lo tanto, en que la osteotomía de falange en el sitio de la deformidad es un procedimiento eficaz para corregir las deformidades de los dedos, con un buen resultado objetivo y subjetivo a mediano plazo. El uso de placas y tornillos determina una tasa más alta de complicaciones, debido a la intolerancia y las adherencias del aparato extensor que estas últimas producen. En consecuencia, se debe advertir a los pacientes sobre la posibilidad de una segunda operación.

Conflicto de intereses: Los autores no declaran conflictos de intereses.

ORCID de L. Ferreira: http:///rcid. org/0000-0003-0356-9510

ORCID de P. De Carli: http:///orcid.org/0000-0002-9474-8129

ORCID de G. L. Gallucci: http://orcid.org/0000-0002-0612-320X
ORCID de A. Donndorff: $h$ ttr. $/ /$ orcid org/0000-0002-6384-4820

ORCID de E. E. Zaidenberg: http://orcid.org/0000-0002-1535-0586

ORCID de J. G. Boretto: http:///orcid.org/0000-0001-7701-3852

\section{BIBLIOGRAFÍA}

1. Lee SG, Jupiter JB. Phalangeal and metacarpal fractures of the hand. Hand Clin 2000;16(3):323-32, vii. PMID: 10955206

2. Büchler U, Gupta A, Ruf S. Corrective osteotomy for post-traumatic malunion of the phalanges in the hand. $J$ Hand Surg Br 1996;21(1):33-42. https://doi.org/10.1016/s0266-7681(96)80009-x

3. Manktelow RT, Mahoney JL. Step osteotomy: a precise rotation osteotomy to correct scissoring deformities of the fingers. Plast Reconstr Surg 1981;68(4):571-6. PMID: 7280104

4. Gross MS, Gelberman RH. Metacarpal rotational osteotomy. J Hand Surg Am 1985;10(1):105-8. https://doi.org/10.1016/s0363-5023(85)80259-8

5. Trumble T, Gilbert M. In situ osteotomy for extra-articular malunion of the proximal phalanx. J Hand Surg Am 1998;23(5):821-6. https://doi.org/10.1016/S0363-5023(98)80156-1

6. Potenza V, De Luna V, Maglione P, Garro L, Farsetti P, Caterini R. Post-traumatic malunion of the proximal phalanx of the finger. Medium-term results in 24 cases treated by "in situ" osteotomy. Open Orthop J 2012. https://doi.org/10.2174/1874325001206010468

7. Botelheiro JC. Overlapping of fingers due to malunion of a phalanx corrected by a metacarpal rotational osteotomy-report of two cases. J Hand Surg Br 1985;10(3):389-90. https://doi.org/10.1016/s0266-7681(85)80070-x

8. Menon J. Correction of rotary malunion of the fingers by metacarpal rotational osteotomy. Orthopedics 1990;13(2):197-200. PMID: 2308879

9. Pieron AP. Correction of rotational malunion of a phalanx by metacarpal osteotomy. J Bone Joint Surg Br 1972;54(3):516-9. https://doi.org/10.1302/0301-620X.54B3.516

10. Gollamudi S, Jones WA. Corrective osteotomy of malunited fractures of phalanges and metacarpals. J Hand Surg Br 2000;25(5):439-41. https://doi.org/10.1054/jhsb.2000.0418

11. Pichora DR, Meyer R, Masear VR. Rotational step-cut osteotomy for treatment of metacarpal and phalangeal malunion. J Hand Surg Am 1991;16(3):551-5. https://doi.org/10.1016/0363-5023(91)90031-6 
12. Dindo D, Demartines N, Clavien P-A. Classification of surgical complications. Ann Surg 2004;240(2):205-13. https://doi.org/10.1097/01.sla.0000133083.54934.ae

13. Tang JB. Re: Levels of experience of surgeons in clinical studies. J Hand Surg Eur Vol. 2009;34(1):137-8. https://doi.org/10.1177/17531934097321

14. van der Lei B, de Jonge J, Robinson PH, Klasen HJ. Correction osteotomies of phalanges and metacarpals for rotational and angular malunion: a long-term follow-up and a review of the literature. J Trauma 1993;35(6):902-8. https://doi.org/10.1097/00005373-199312000-00017

15. Balaram AK, Bednar MS. Complications after the fractures of metacarpal and phalanges. Hand Clin 2010;26(2):169-77. https://doi.org/10.1016/j.hcl.2010.01.005

16. Gajendran VK, Gajendran VK, Malone KJ. Management of complications with hand fractures. Hand Clin 2015;31(2):165-77. https://doi.org/10.1016/j.hcl.2014.12.001

17. Ataker Y, Uludag S, Ece SC, Gudemez E. Early active motion after rigid internal fixation of unstable extra-articular fractures of the proximal phalanx. Hand Surg Eur Vol 2017;42(8):803-9. https://doi.org/10.1177/1753193417709949 